\title{
The Improved Ant Colony Optimization Algorithm for MLP considering the Advantage from Relationship
}

\author{
Yabo Luo and Yongo P. Waden \\ School of Mechanical and Electronic Engineering, Wuhan University of Technology, Wuhan City 430070, China \\ Correspondence should be addressed to Yabo Luo; luoyabo1973@163.com
}

Received 27 December 2016; Revised 27 March 2017; Accepted 4 June 2017; Published 31 July 2017

Academic Editor: Gen Q. Xu

Copyright (c) 2017 Yabo Luo and Yongo P. Waden. This is an open access article distributed under the Creative Commons Attribution License, which permits unrestricted use, distribution, and reproduction in any medium, provided the original work is properly cited.

\begin{abstract}
An improved ant colony optimization (ACO) is presented to solve the machine layout problem (MLP), and the concept is categorized as follows: firstly, an ideology on "advantage from quantity" and "advantage from relationship" is proposed and an example is demonstrated. In addition, the strategy of attached variables under local polar coordinate systems is employed to maintain search efficiency, that is, "advantage from relationship"; thus, a mathematical model is formulated under a single rectangular coordinate system in which the relative distance and azimuth between machines are taken as attached design variables. Further, the aforementioned strategies are adopted into the ant colony optimization (ACO) algorithm, thereby employing the inverse feedback mechanism for dissemination of pheromone and the positive feedback mechanism for pheromone concentration. Finally, the effectiveness of the proposed improved ACO is tested through comparative experiments, in which the results have shown both the reliability of convergence and the improvement in optimization degree of solutions.
\end{abstract}

\section{Introductions}

A machine layout problem (MLP) is categorized under a quadratic assignment problem (QAP) which is known as NPhard and unsolvable with traditional optimization methods $[1,2]$. Thus, bionic algorithms which mimic the principles or behaviors of living organisms to achieve optimization in an objective function, have achieved some progress in solving MLP $[3,4]$. For instance, Samarghandi and Eshghi [5] proposed a new method based on tabu search for a single row facility layout problem (SRFLP) and they have reported better solutions than other methods in the literature. Further, Selvakumar et al. were able to optimize the equipment layout by employing an improved artificial neural network [6]. Meanwhile, GA improvements, such as in the permutationbased [7] and the reverse constraint satisfied tactics [8], have been applied to determine the optimal solutions as well. Further, the application of swarm intelligence such as artificial bee colony (ABC) [9-11], Particle Swarm Optimization (PSO) $[12,13]$, and the ACO $[14,15]$ have contributed significantly to solving combinatorial problems. Nevertheless, literature is widely dominated by applications of the swarm intelligence in tackling MLP, especially, the ACO [16]. Ulutas and KulturelKonak [17] have indicated that research in MLP is most significant and fruitful.

Notwithstanding all the progress in solving MLP, however, majority of the research concentrates on the smallsize and standardized problems, which are mainly focused on linear placement of rectangular facilities with varying dimensions on some straight lines such as the single row [18] and double row layout [19]. Although few researchers tried to solve the multiline and flexible layout problems [20, 21], search efficiency and convergence cannot be guaranteed. As pointed out in [22] satisfactory solutions to large-scale problems are difficult to obtain. In addition, as far as the literature is concerned, no research existed on exploring the reasons of inefficiency in convergence and search in the large instance of MLP, except some attempts by Drira et al. [23] and Saravanan and Arulkumar [24]. Therefore, research on MLP under a flexible manufacturing environment with minimum constraints is still in a primary stage. As based on the analysis of the key reasons for the inefficiency of search and poor 
convergence, this research proposes a novel approach that transforms the coordinate system for design variables and constraint space to local polar coordinate systems. Thereby, ACO algorithm is improved by incorporating the novel approach, in order to optimize the problem of inefficiency in convergence and search strategy in the ACO.

\section{2. "Advantage from Quantity" and "Advantage from Relationship"}

From optimization perception, two kinds of advantages, named "advantage from quantity" and "advantage from relationship" [25], are constructed based on natural behaviors in insects, animal, or human, and, as the names sound, no such strategy has been reported in the wide literature of computational intelligence.

Advantage from Quantity. It means an advantage usually comes from the quantity of a certain property, which is contingent on the measurement of a certain value. For example, the loading capacity of a lorry depends on its volume; the viability of a giraffe depends on the length of its neck; the survival chance of a locust depends on its similarity in color with grass. Consequently, these examples, the volume, length, and the degree of a certain property are all advantages from quantity.

Advantage from Relationship. It is from a relationship, which is not contingent on the independent features of properties but on the relationship among them; for example, the equilibrium of a plane lies not on the independent loading capacity of the front cabin, middle cabin, or the rear cabin but on proportion of them, and the balance of a dragonfly lies not in the weight of the head or the independent value of the length of torso but in the ratio between them. Therefore, from these examples, advantages are contingent on the relationship among properties and, thus, belong to the advantage from relationship.

Furthermore, the major difference between the two kinds of advantages is that the advantage from quantity is convergent in a normal bionic algorithm whereas the advantage from relationship is not, because distribution of values in the feasible regions of these two advantages is quite different. Hence, the differences can be illustrated by examples as follows.

Example 1. Let us determine the values of $x, y$, and $z$ :

$$
\begin{aligned}
\operatorname{Max} & F=x+y+z \\
\text { s.t. } & x, y, z \in[0,15] .
\end{aligned}
$$

From the genetic algorithm, binary design variables are defined as follows:

$B$

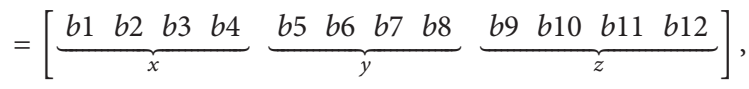

where $B=\left[\begin{array}{llllllllllll}1 & 1 & 1 & 1 & 0 & 1 & 1 & 1 & 0 & 1 & 0 & 1\end{array}\right]$, and then $x=$ $15, y=7$, and $z=5$

If the coding method above is adopted and run in 10 iterations, numerous individuals in the population can be updated as $B=\left[\begin{array}{llllllllllll}1 & 1 & 1 & 1 & 1 & 1 & 1 & 1 & 1 & 1 & 1 & 1\end{array}\right]$, whereby the optimal individuals are $x=15, y=15$, and $z=$ 15. Further, if the coding is run 20 times of iterations, all individuals are updated to their optimal position and also stability is maintained.

Example 2. Let us find the values of $x, y$, and $z$ where

$$
\begin{gathered}
x=y \times z \\
\text { s.t: } \quad x, y, z \in[0,15] .
\end{gathered}
$$

Again if genetic algorithm principle is adopted, the search efficiency is low, because of the variant factors involved; additionally, the optimal population cannot be maintained because the solution space takes on a status of divergence in further iterations.

The reason for difference can be seen in Example 1, a problem with "advantage from quantity" which has a feasible region with a good property though is monotonic. Moreover, the population formed by fit individuals that are more capable of generating fitted offspring, as such, is shown by the following.

Let us assume that

$$
\begin{aligned}
& B 1=\left[\begin{array}{llllllllllll}
1 & 1 & 1 & 0 & 1 & 0 & 1 & 1 & 1 & 1 & 0 & 1
\end{array}\right], \\
& B 2=14, y=11, z=13, \\
& B 3=\left[\begin{array}{llllllllllll}
1 & 0 & 1 & 1 & 0 & 1 & 1 & 0 & 1 & 1 & 1 & 0
\end{array}\right], \\
& \left.\begin{array}{llllllllllll}
1 & 0 & 1 & 0 & 0 & 1 & 1 & 1 & 1 & 0 & 0 & 0
\end{array}\right], \\
& B 4=\left[\begin{array}{llllllllllll}
1 & 0 & 0 & 1 & 0 & 0 & 1 & 0 & 1 & 0 & 1 & 0
\end{array}\right], y=6, z=14, \\
&
\end{aligned}
$$

Hence, from (4), individuals $B 1$ and $B 2$ are better than $B 3$ and $B 4$, where their offspring have high possibility of achieving better fitness compared to those from B3 and B4. Therefore, the search process of problem with "advantage from quantity" is convergent and the optimized population is stable. On the contrary, as is seen in Example 2, a problem with "advantage from relationship," where the offspring produced by fitted parents are not better than those produced by random individuals in the feasible region, is shown below:

$$
\begin{aligned}
& B 1=\left[\begin{array}{llllllllllll}
0 & 1 & 1 & 0 & 0 & 0 & 1 & 0 & 0 & 0 & 1 & 1
\end{array}\right], \\
& \quad x=6 \quad y=2 z=3, \\
& B 2=\left[\begin{array}{llllllllllll}
1 & 1 & 0 & 0 & 0 & 0 & 1 & 1 & 0 & 1 & 0 & 0
\end{array}\right], \\
& \\
& x=12 y=3 z=4,
\end{aligned}
$$




$$
\begin{aligned}
& B 3=\left[\begin{array}{llllllllllll}
1 & 0 & 0 & 0 & 0 & 0 & 1 & 1 & 0 & 1 & 0 & 0
\end{array}\right], \\
& x=8 \quad y=3 z=4, \\
& B 4=\left[\begin{array}{llllllllllll}
1 & 0 & 0 & 1 & 0 & 0 & 1 & 0 & 0 & 0 & 1 & 1
\end{array}\right] \text {, } \\
& x=9 y=2 z=3 \text {. }
\end{aligned}
$$

The individuals $B 1$ and $B 2$ are optimal; meanwhile, individuals $B 3$ and $B 4$ are not optimal, but, after crossover operations, individuals updated by $B 1$ and $B 2$ are not optimal whereas those updated by $B 3$ and $B 4$ are likely to be optimal individuals. In addition, for a population formed by fit individuals, those having "advantage from relationship" do not necessarily have to be fit to generate fit offspring, hence, that can lead to inefficiency in convergence and search process.

A machine layout problem (MLP) is a typical problem, with "advantage from relationship." That is, the efficiency of materials flow (logistic) in the machines is not contingent on the independent locations of each machine but on the relationship among them. Further, inefficiency in logistics can be realized still, if a rectangular coordinate system is considered for the logistic in MLP, where the coordinates of locations for machines are changed but relative positions relationships are maintained. On the contrary, efficiency in logistics can be increased if the locations of few machines are changed; meanwhile, other locations of machines are kept still. Therefore, the interaction between the locations of machines and the efficiency in logistics cannot be illustrated by considering rectangular coordinates of machines as design variables only but also by controlling the design variables in order to illustrate the relationship of the relative positions of machines.

\section{Basic MLP Model under Rectangular Coordinate System}

A MLP can be described as follows: $n$ machines are arranged in a workshop with the dimensions such as length $(L)$ and width $(W)$. And each geometry of a machine is denoted by a rectangular box whereby $L$ and $W$ are represented by $S_{i}$ and $Q_{i}$, respectively. In addition, the objective function is a minimization of the total cost of material flow in the machines under geometric constraint of the workspace, where the geometric description of workshop is shown in Figure 1.

The optimization model for minimization of the total cost for material flow (logistic) can be described mathematically as follows:

$$
\begin{array}{ll}
\min & F=\sum_{i=1}^{n} \sum_{j=1}^{n} f_{i j} \times d_{i j} \times c_{i j} \\
\text { s.t. } & d_{i j}=\sqrt{\left(x_{i}-x_{j}\right)^{2}+\left(y_{i}-y_{j}\right)^{2}} .
\end{array}
$$

Equation (7) describes the logistic distance between machines $i$ and $j$ :

$$
f=\left[\begin{array}{cccc}
f_{11} & f_{12} & \cdots & f_{1 n} \\
f_{21} & f_{22} & \cdots & f_{2 n} \\
\vdots & \vdots & \cdots & \vdots \\
f_{n 1} & f_{n 2} & \cdots & f_{n n}
\end{array}\right] \quad i=1, \ldots, n ; j=1, \ldots, n .
$$

Equation (8) is for logistic frequency matrix among machines:

$$
d=\left[\begin{array}{cccc}
d_{11} & d_{12} & \cdots & d_{1 n} \\
d_{21} & d_{22} & \cdots & d_{2 n} \\
\vdots & \vdots & \cdots & \vdots \\
d_{n 1} & d_{n 2} & \cdots & d_{n n}
\end{array}\right]
$$

Equation (9) is for logistic distance matrix among machines:

$$
c=\left[\begin{array}{cccc}
c_{11} & c_{12} & \cdots & c_{1 n} \\
c_{21} & c_{22} & \cdots & c_{2 n} \\
\vdots & \vdots & \cdots & \vdots \\
c_{n 1} & c_{n 2} & \cdots & c_{n n}
\end{array}\right] .
$$

Equation (10) is used to determine the unit-distance logistic cost matrix among machines:

$$
\begin{aligned}
& x_{i}+\frac{S_{i}}{2}<L \\
& \frac{S_{i}}{2}-x_{i}<0 \\
& \quad \text { for } i=1, n, \\
& y_{i}+\frac{Q_{i}}{2}<W \\
& \frac{Q_{i}}{2}-y_{i}<0 \\
& \quad \text { for } i=1, n .
\end{aligned}
$$

Equation (12) is devised for boundary constraints of length and width in the geometric space:

$$
\begin{aligned}
& \text { for } i=1, n\left\{\begin{array}{l}
\text { for } j=i, n\left\{\left|x_{i}-x_{j}\right| \geq\left|S_{i}+S_{j}\right| \text { OR }\left|x_{i}-x_{j}\right| \geq\left|S_{i}+S_{j}\right|\right\} \\
\} \\
S_{i}=S_{i}+R_{i S} \\
Q_{i}=Q_{i}+R_{i Q} .
\end{array}\right.
\end{aligned}
$$

Meanwhile, Equations (13)-(14) are for interference and tolerance constraints, respectively, where the tolerance zones are represented by $R_{i S}$ and $R_{i Q}$.

Therefore, MLP is a typical example of a problem with "advantage from relationship," where the location of a certain single machine makes no meaning to the optimization of a layout, as the logistic efficiency is determined by relative position relationship among machines. In addition, if a mathematical model is formulated based on independent position coordinates of machines, that is, design variables, the reliability and efficiency of the search process will be poor still, and, in order to improve the efficiency and search reliability, a technique of controlling design variables under 


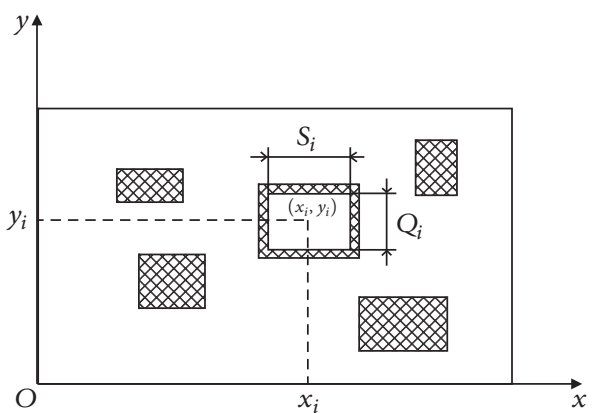

FIGURE 1: The geometric description of machine layout problem.

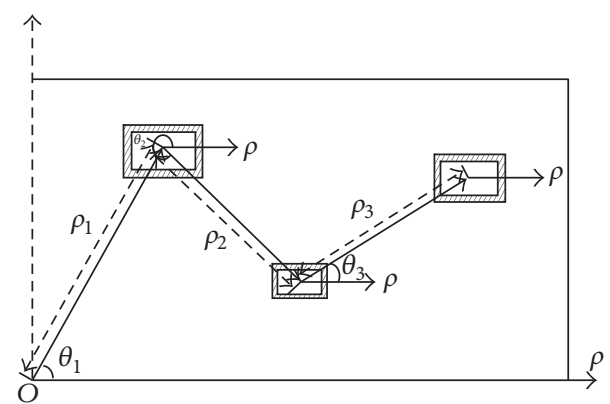

FIGURE 2: Description of the attached position relationship of machines.

local polar coordinate systems is proposed; consequently, a correlation between design variables and objective function is effectively demonstrated.

\section{The Attached Design Variables and Mathematical Model under Local Polar Coordinate Systems}

In this section, the design variables and mathematical model which influence the performance of ACO are constructed under polar coordinates. Moreover, main objective is improvement by considering a relative position relationship of machines. Firstly, a transformation of the rectangular coordinate geometry into a series of local polar coordinate systems is devised as depicted in Figure 2, in which the first local polar coordinate system is set as in Figure 1; thus, every geometric center of machines is set as a local polar origin, and their local polar axes are directed as $x$-axis.

From Figure 2, the search strategy of the ant colony under local polar coordinate systems can be explained as follows: first, let us assume the machines are in any numbered permutation and say if the location of first machine is determined then the search will automatically go to the next machine that follows, in which search is determine by the azimuth and relative distance of the machines. Additionally, search on a path is completed in a stepwise procedure. Intuitively, design variables and the objective function are significantly correlated. Moreover, fit individuals have high probability to produced fit offspring; as a consequence, the "advantage from relationship" can be maintained in any iteration. Hence, the design variables and objective function can be defined as follows:

$$
\begin{aligned}
\text { Determine: } X & =\left(\rho_{1}, \theta_{1}, \rho_{2}, \theta_{2}, \ldots, \rho_{n}, \theta_{n}\right) \\
\min F & =\sum_{i=1}^{n-1} \sum_{j=i+1}^{n} f_{i j} \times d_{i j} \times c_{i j}
\end{aligned}
$$

To clarify more on the position relationship where the distance between two machines under polar coordinate is to be determined, let us assume $n$ machines are denoted as 1,2 , 3 , and 4 as depicted in Figure 3. The attached design variables, key parameter $d_{i j}$, boundary constraint, and the interference free constraint can be expressed mathematically as follows:

$$
\begin{aligned}
& A D=\rho_{2} \cos \theta_{2}+\rho_{3} \cos \theta_{3}+\rho_{4} \cos \theta_{4} \\
& E D=\rho_{2} \sin \theta_{2}+\rho_{3} \sin \theta_{3}+\rho_{4} \sin \theta_{4}
\end{aligned}
$$

where

$$
d_{14}=\sqrt{A D^{2}+E D^{2}}
$$

where the formula for calculating distances between machines at positions $i$ and $j$ is

$$
d_{i j}=\sqrt{\left(\rho_{i+1} \cos \theta_{i+1}+\rho_{i+2} \cos \theta_{i+2}+\cdots+\rho_{j} \cos \theta_{j}\right)^{2}+\left(\rho_{i+1} \sin \theta_{i+1}+\rho_{i+2} \sin \theta_{i+2}+\cdots+\rho_{j} \sin \theta_{j}\right)^{2}} .
$$

Further, expressions that can maintain boundary constraints in abscissas and ordinates of all machines are as follows.

Abscissa

$$
\begin{aligned}
x & =x+\rho_{i} \cos \theta_{i} \quad i=1, \ldots, n \\
x^{*} & =x+\frac{s_{i}}{2}+R_{i s} \quad x^{*}<L,
\end{aligned}
$$

where $x$ is the sum of center for abscissa, $s_{i}$ is the length of machine, and $R_{i s}$ is the tolerance zone.

\section{Ordinate}

$$
\begin{gathered}
y=y+\rho_{i} \sin \theta_{i} \quad i=1, \ldots, n \\
y^{*}=y+\frac{Q_{i}}{2}+R_{i Q} \quad y^{*}<W,
\end{gathered}
$$

where $y$ is sum of the ordinate centers and $Q_{i}$ is the width of machine and $R_{i Q}$ the tolerance zone

$$
\frac{\left(S_{i}+S_{i+1}\right)}{2}+R_{i s}+R_{(i+1) s} \leq \rho_{i+1} \cos \theta_{i+1}
$$




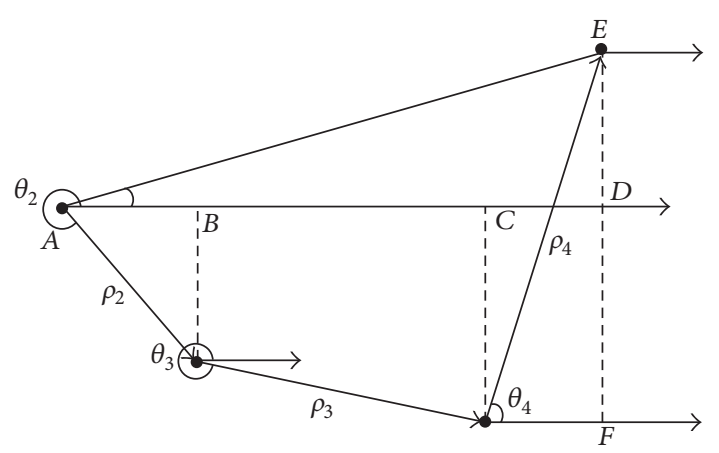

Figure 3: The geometric description of the distance between machines.

$$
\begin{aligned}
\frac{\left(Q_{i}+Q_{i+1}\right)}{2}+R_{i Q}+R_{(i+1) Q} \leq \rho_{i+1} \sin \theta_{i+1} & \\
& i=1, \ldots, n .
\end{aligned}
$$

Equations (20) and (21) are for interference-free constraint among machines.

\section{An Improved ACO Algorithm Based on Attached Design Variables under Local Polar Coordinate Systems}

The perceptive unit of a regular ACO algorithm is the small neighborhood around the ant, and here the position relationship of two close ordinal machines is taken as the perceptive unit whereby a search mechanism can be seen as in Figure 4, in which each machine is considered to be a node for state transition and the position relationship between two close ordinal machines as the perceptive unit of an ant.

Furthermore, an ant is considered to move along the coordinates to disseminate pheromone, and thereby the location of a machine is determined based on the movement. Additionally, negative feedback mechanism occurs after an ant has disseminated pheromone, and also the concentration of the pheromone is perceived using the positive feedback mechanism.

With the fact that design variables $\rho$ and $\theta$ are interconnected, decision on their property is not made on one variable. For instance, for a different value of $\theta$, the property of design variables can vary, even though the value of $\rho$ is kept the same. Therefore, the neighborhood of the state nodes is taken as the perceptive area of disseminated pheromone in order to assure the optimized region sustains the property of "advantage from relationship," which is shown as in Figure 5.

Different from regular ACO algorithm, the dissemination of pheromone in this research adopts the inverse feedback mechanism: ants do not disseminate pheromone in the process of search; in addition, the pheromone is updated only after finding the whole path for the machine layout is searched and pheromone is given to the neighbor node by using formula (22) which combines the objective function

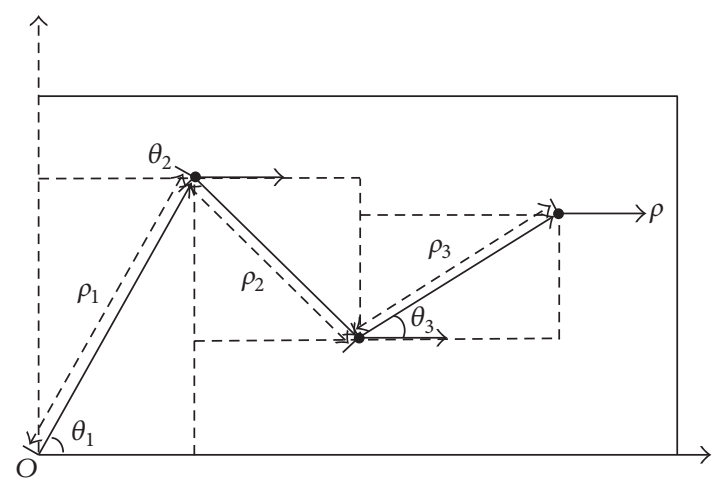

FIgURE 4: Ant perceptive unit model based on attached design variables under local polar coordinate systems.

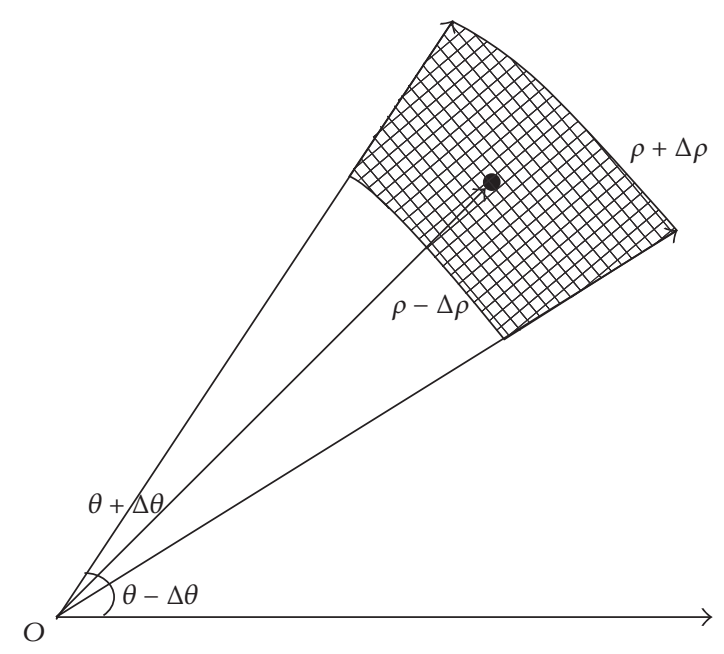

FIGURE 5: Region where ants perceive and disseminate pheromone.

and a constraint, and also $f$ in (22) lies in shaded area of the graph as in Figure 5.

$$
f=\frac{K}{F \times \lambda_{1} \times e^{F_{1}+F_{2}}+\lambda_{2} \times 1 /\left(\left|F_{3}+F_{4}\right| \times \eta+\varepsilon\right)} .
$$

In formula (22), $f$ is a pheromone value for the path in a neighbor node and is assigned inversely, whereas $K$ is a constant; $\lambda_{1}$ and $\lambda_{2}$ are penalty coefficients, and these penalty coefficients take effect when an ant is outside the geometry of the workshop. Meanwhile, $F$ is the value of an objective function and if $F$ is high, then logistic efficiency is low; thus, few pheromones are assigned to the path in neighbor nodes. Further, $F_{1}$ and $F_{2}$ are penalty terms of boundary constraints which equal zero if machines are within the boundary. Otherwise, $F_{1}$ and $F_{2}$ will be a positive value. The mathematical expressions are given as follows:

$$
\begin{aligned}
x & =x+\rho_{i} \cos \theta_{i} \quad i=1, \ldots, n, \\
y & =y+\rho_{i} \sin \theta_{i}, \\
F_{1} & =F_{1}+\left(\frac{\left|x+S_{i} / 2-L\right|+x+S_{i} / 2-L}{2}\right)^{2}
\end{aligned}
$$




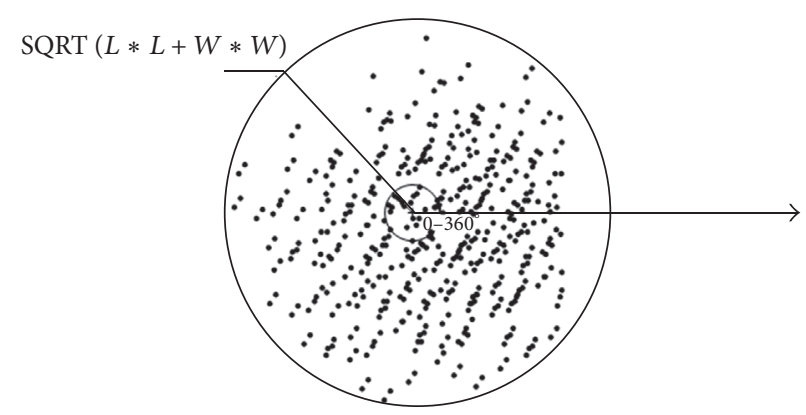

FiguRE 6: The accumulative pheromone space of a machine under local polar coordinate system.

$$
\begin{gathered}
+\left(\frac{\left|S_{i} / 2-x\right|+S_{i} / 2-x}{2}\right)^{2} \\
F_{2}=F_{2}+\left(\frac{\left|y+Q_{i} / 2-W\right|+y+Q_{i} / 2-W}{2}\right)^{2} \\
+\left(\frac{\left|Q_{i} / 2-y\right|+Q_{i} / 2-y}{2}\right)^{2}
\end{gathered}
$$

where $F_{3}$ and $F_{4}$ are penalty terms for overlap interference constraint. If there is no overlap interference among machines, $F_{3}$ and $F_{4}$ equal zero and their values are calculated by

$$
\begin{aligned}
x & =x+\rho_{i} \cos \theta_{i} \quad i=1, \ldots, n \\
y & =y+\rho_{i} \sin \theta_{i} \\
F_{3} & =\left|\left(\left|x_{i}-x_{j}\right|-S_{i}-S_{j}\right)\right|+\left|x_{i}-x_{j}\right|-S_{i}-S_{j} \\
F_{4} & =\left|\left(\left|y_{i}-y_{j}\right|-Q_{i}-Q_{j}\right)\right|+\left|y_{i}-y_{j}\right|-Q_{i}-Q_{j},
\end{aligned}
$$

where, in (22), $\eta$ is set to a large number and $\varepsilon$ to a small number. If overlap is experienced then interferences $F_{3}$ and $F_{4}$ are equal to zero, $\eta$ is redundant, and the reciprocal of $\varepsilon$ is a large value, and thus, the penalty function works. In case of no overlap, interferences $F_{3}$ and $F_{4}$ are not equal to zero, and $\eta$ is a large value. Hence, the reciprocal of penalty term is rather small; therefore, the penalty function does not work. After the completion of the whole path for machine layout is searched, the disseminated pheromone in the neighborhood of every machine is placed under local polar coordinate. Thereby, an accumulative pheromone space is located in a polar angle of $[0,2 \pi]$ and polar radius $\left[0, \sqrt{L^{2}+W^{2}}\right]$ which are under a polar coordinate system. In addition, because the length of the diagonal is the longest linear distance in the workshop, the maximum value of polar radius cannot exceed $\sqrt{L^{2}+W^{2}}$ and is graphically depicted in Figure 6 in which the maximum distance an ant must cover under the polar coordinates which corresponds to the geometric movement of the ant under polar coordinates as shown in Figures 4 and 5.

The positive feedback mechanism is adopted to perceive pheromone concentration, whereby machines are considered as nodes; each time the ant passes a node, the pheromone of that node is distributed under the local polar coordinate system and, hence, is used as a probability condition to choose a relative location of a next node. Thus, the pheromone concentration of point in any local polar coordinate system is calculated by

$$
p_{\theta \rho}=\frac{\tau_{\theta \rho}}{\int_{\theta=0}^{2 \pi} \int_{\rho=0}^{\sqrt{L^{2}+W^{2}}} \tau_{\theta \rho}},
$$

where $p_{\theta \rho}$ is the pheromone concentration and $\tau_{\theta \rho}$ is the pheromone value of a certain point all under the local polar coordinate. The selection criterion for a machine (node) placement is based on the probability given by (25), which is under a local polar coordinate. Meanwhile, a minimum error of probability is set and a random point is selected as the placement point. Therefore, from the two strategies aforementioned the placement of any machine will have the following three characteristics:

(1) The location of a machine is considered based on the relative location relationship between the predecessor and the successor machine, and that can reflect the relevancy between design variables and the advantage from relationship.

(2) The node with a high concentration of pheromone has a high probability to be chosen as next placement point.

(3) The node with a low concentration of pheromone is not necessarily excluded from the placement point and only has low probability to be selected. Hence, a minimum error of probability exists during a node selection. These conditions are preserved to avoid local optimum solution.

Based on the strategy of local polar coordinate system, pheromone inverse feedback disseminating mechanism and the positive feedback perceiving mechanism mentioned before result in an improvement of the ACO algorithm.

\section{Illustrative Example}

In order to illustrate the superiority of improved ACO algorithm under local polar coordinate system, a comparative experiment is performed between GA and ACO and 


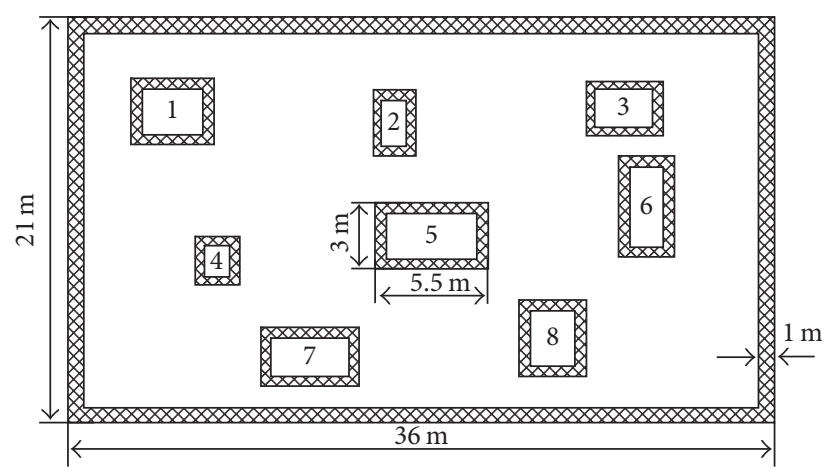

FIgURE 7: The physical dimension of the case.

improved ACO are considered under rectangular coordinate system.

The data considered in this research have been used by many researchers; thus, numerous experiments have been carried out, and, as a consequence, the results found are good. Further, considering the length of the paper, only one of the cases is detailed in this paper. The case is as follows: eight machines are arranged into a workshop with a length of 36 meters and width of 21 meters as shown in Figure 7. The 1 meter is kept as peripheral length, where all machines are kept away from the workshop walls. In addition, the distance of geometric centers of any two machines is required to be no less than 6 meters. The geometry values for machines placement including tolerance zones are calculated by (26). The unit-distance material flow (logistic) cost matrix is determined by (27) and the logistic frequency among machines is calculated by (28).

$$
\begin{aligned}
G & =\left[\begin{array}{ll}
S & Q
\end{array}\right] \\
& =\left[\begin{array}{llllllll}
5.0 & 2.0 & 3.0 & 2.0 & 5.5 & 2.0 & 5.5 & 3.5 \\
2.5 & 3.0 & 1.5 & 2.0 & 3.0 & 5.0 & 2.5 & 4.0
\end{array}\right], \\
c_{i j} & =\left[\begin{array}{cccccccc}
0 & 1.2 & 1.8 & 2.5 & 1.2 & 2.3 & 2.1 & 2.6 \\
1.2 & 0 & 1.5 & 2.0 & 1.8 & 2.0 & 1.5 & 1.6 \\
1.8 & 1.5 & 0 & 2.2 & 1.6 & 1.2 & 1.0 & 2.2 \\
2.5 & 2.0 & 2.2 & 0 & 2.5 & 2.4 & 3.2 & 1.0 \\
1.2 & 1.8 & 1.6 & 2.5 & 0 & 2.1 & 2.3 & 3.0 \\
2.3 & 2.0 & 1.2 & 2.4 & 2.1 & 0 & 1.5 & 2.5 \\
2.1 & 1.5 & 1.0 & 3.2 & 2.3 & 1.5 & 0 & 1.2 \\
2.6 & 1.6 & 2.2 & 1.0 & 3.0 & 2.5 & 1.2 & 0
\end{array}\right], \\
f_{i j} & =\left[\begin{array}{ccccccccc}
0 & 21 & 28 & 23 & 35 & 22 & 32 & 35 \\
21 & 0 & 55 & 24 & 25 & 30 & 22 & 18 \\
28 & 55 & 0 & 34 & 60 & 45 & 25 & 33 \\
23 & 24 & 34 & 0 & 30 & 25 & 30 & 40 \\
35 & 25 & 60 & 30 & 0 & 18 & 28 & 15 \\
22 & 30 & 45 & 25 & 18 & 0 & 21 & 22 \\
32 & 22 & 25 & 30 & 28 & 21 & 0 & 41 \\
35 & 18 & 33 & 40 & 15 & 22 & 41 & 0
\end{array}\right] .
\end{aligned}
$$

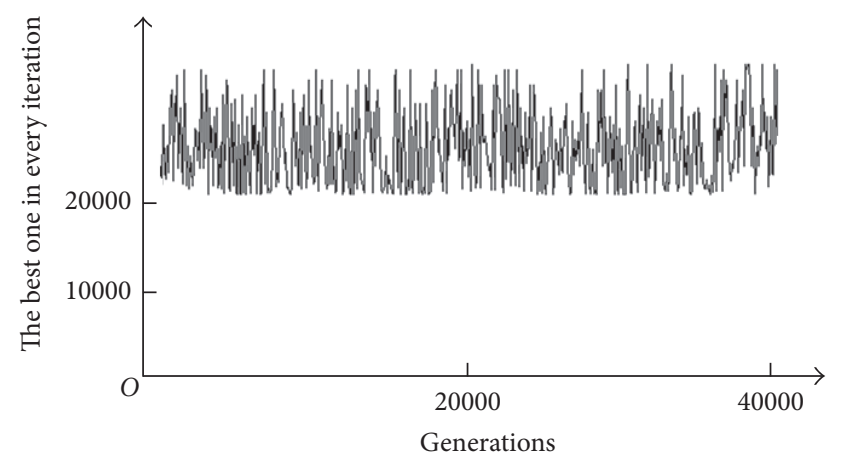

FIGURE 8: The curve of iterations adopting genetic algorithm under rectangular coordinate system.

The experiment for layout is performed by considering generations as the abscissa and logistic cost $F$ as the ordinate. Additionally, the values of logistic cost $F$ and design variables for the optimal individuals in different generations are shown in Table 1. The experimental results of 40000 generations are shown in Figure 8, where genetic algorithm is adopted under rectangular coordinate system. As is seen from Figure 8 after 40000 iterations, there is no sign of convergence and the $F$ value in population fluctuates from around 20000 to 30000 . Further, other considerations were made such as cross factors are set from 0.2 to 0.9 and variation factor is set from 0.02 to 0.2 ; however, the same results are obtained. It is worth mentioning that the best fitness is maintained during the variations of the cross factors and variation factor. Therefore, it is concluded that when the problem has no less than 8 machines, the iteration process is not convergent for a genetic algorithm under rectangular coordinate system.

In Tables 1 and 2, the results obtained by employing GA and regular ACO, respectively, under polar coordinates are shown. The $x$ and $y$ values are for the local optimal value in which a machine layout is to be located, whereas the $F$ value is the cost of material flow (logistic flow) in the machines. And, from these results in Tables 1 and 2, as is seen $F$ values are increasing or fluctuating within a trapped local optimal value as the number of generations is increased, and also more evidence on that is depicted in Figure 9. However, as can be seen in Table 3, the convergence results for the improved ACO are better as can be noticed in $F$ values, when the number of generations is increased.

Furthermore, an experiment is conducted by employing the regular ACO algorithm under rectangular coordinate system, and it was noticeable that the trait is trapped into the local optimal value. However, several experiments are performed and only after 500 iterations is the optimal search found in the local optimal value which is even far from the global solution. The search trait can be seen in Figure 9. In addition, some local optimal value which are obtained from numerous searches are reported in Table 2.

When the improved ACO was run under the same conditions of GA and ACO, in the 750 iterations the optimal-seeking appears to be convergent and finally optimal solution is reached in around 900 iterations. Hence, the 
TABLE 1: Results of genetic algorithm under rectangular coordinate system.

\begin{tabular}{|c|c|c|c|c|c|c|c|c|c|}
\hline \multirow{2}{*}{ Generations } & $x_{1}$ & $x_{2}$ & $x_{3}$ & $x_{4}$ & $x_{5}$ & $x_{6}$ & $x_{7}$ & $x_{8}$ & \multirow[t]{2}{*}{$F$} \\
\hline & $y_{1}$ & $y_{2}$ & $y_{3}$ & $y_{4}$ & $y_{5}$ & $y_{6}$ & $y_{7}$ & $y_{8}$ & \\
\hline \multirow{2}{*}{300} & 4.24 & 10.48 & 6.39 & 17.26 & 23.49 & 27.74 & 19.90 & 23.66 & \multirow{2}{*}{22282} \\
\hline & 4.30 & 7.42 & 15.44 & 13.72 & 10.54 & 14.78 & 8.13 & 4.95 & \\
\hline \multirow{2}{*}{5000} & 20.03 & 9.04 & 5.02 & 19.29 & 27.34 & 26.40 & 16.99 & 29.94 & \multirow{2}{*}{21851} \\
\hline & 16.57 & 16.92 & 4.55 & 9.19 & 5.18 & 15.13 & 2.19 & 11.59 & \\
\hline \multirow{2}{*}{15000} & 30.68 & 5.69 & 14.59 & 12.25 & 28.06 & 22.10 & 6.73 & 18.14 & \multirow{2}{*}{23019} \\
\hline & 12.15 & 12.93 & 3.56 & 18.27 & 2.98 & 16.74 & 5.58 & 9.28 & \\
\hline \multirow{2}{*}{20000} & 22.28 & 28.06 & 22.62 & 29.27 & 6.16 & 9.34 & 17.11 & 13.41 & \multirow{2}{*}{20736} \\
\hline & 11.35 & 15.28 & 4.59 & 6.76 & 16.28 & 8.94 & 16.72 & 5.31 & \\
\hline \multirow{2}{*}{30000} & 32.99 & 31.54 & 5.54 & 6.61 & 17.72 & 26.01 & 6.80 & 26.72 & \multirow{2}{*}{26658} \\
\hline & 4.27 & 18.72 & 15.68 & 4.46 & 8.85 & 1.06 & 0.46 & 9.83 & \\
\hline \multirow{2}{*}{40000} & 6.81 & 12.60 & 7.15 & 13.81 & 22.95 & 30.23 & 18.27 & 31.21 & \multirow{2}{*}{22306} \\
\hline & 13.37 & 17.30 & 6.61 & 8.77 & 14.88 & 6.63 & 5.69 & 16.72 & \\
\hline
\end{tabular}

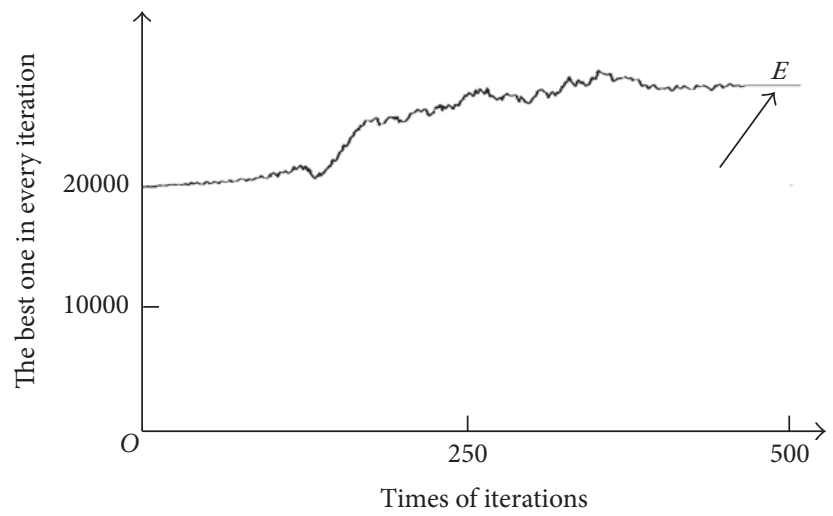

FIGURE 9: The curve of searching adopting regular ACO algorithm under rectangular coordinate system.

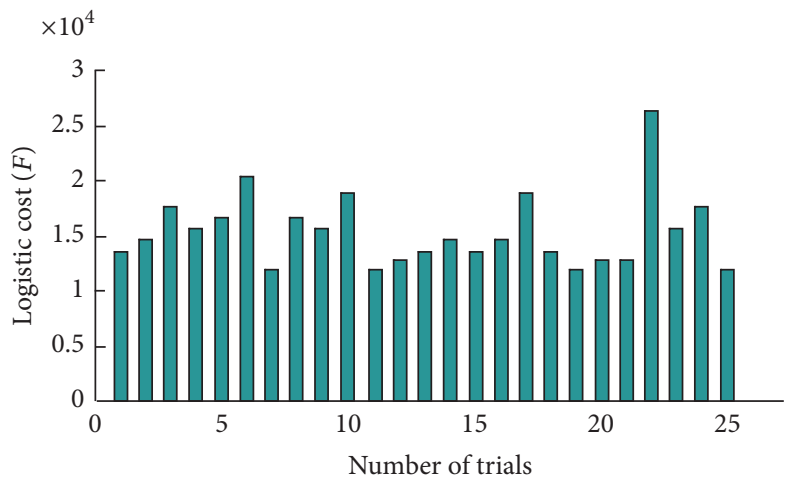

FIGURE 10: Convergence of an improved ACO placed under Cartesian polar coordinates.

optimal-seeking results of critical iterations are shown in Table 3. As can be seen, after 916 iterations, the optimal solution is obtained which corresponds to the logistic cost 11768. Figure 10 shows reliability results for 25 independent runs executed by the improved ACO algorithm under Cartesian coordinates. Moreover, as can be seen in Figure 11, the infeasible layout during critical iterations is observed.
Further, every machine location has impact on logistic cost; thus, in Figure 11 locations of the machines are different in the layouts; for instance, location of machine 5 in 100 iterations (Figure 11(a)) is different from that in 200 iterations and that supposedly is to change the logistic cost $(F)$ for the layout when convergence is feasible. Moreover, placement of machine is based on ant movement as depicted in 


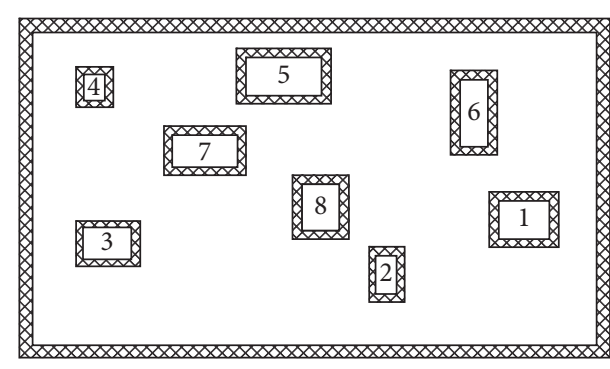

100 times of iterations

(a)

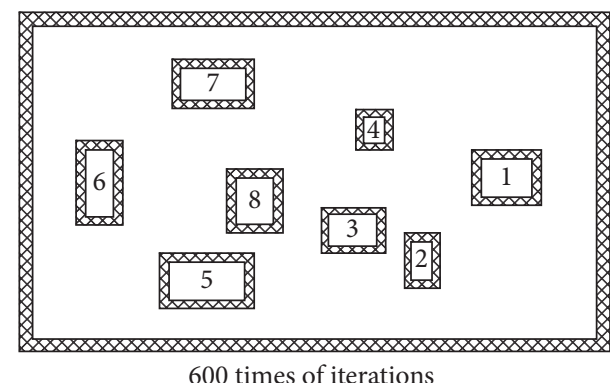

600 times of iterations

(c)

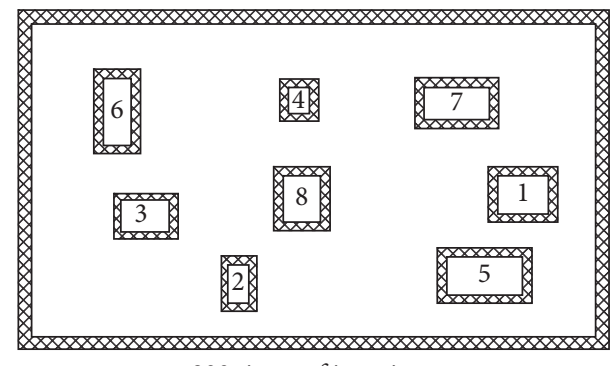
800 times of iterations

(e)

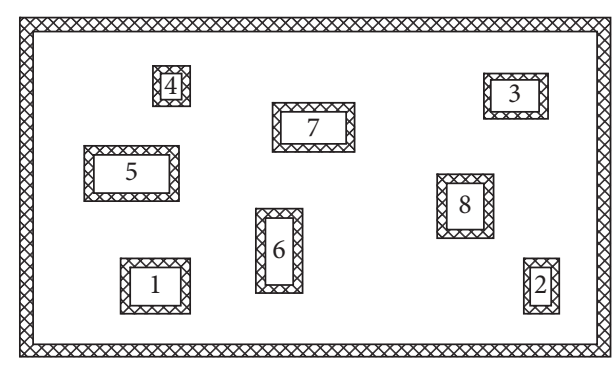

200 times of iterations

(b)

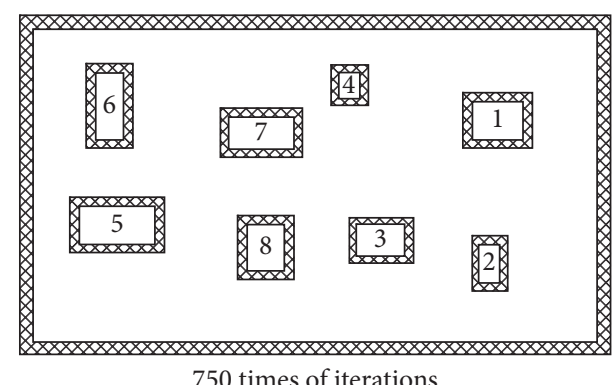

750 times of iterations

(d)

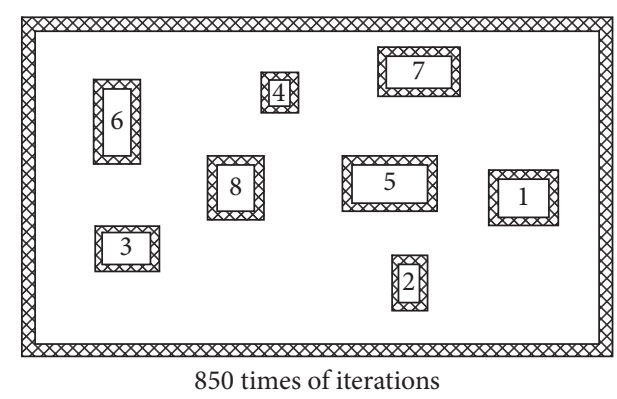

(f)

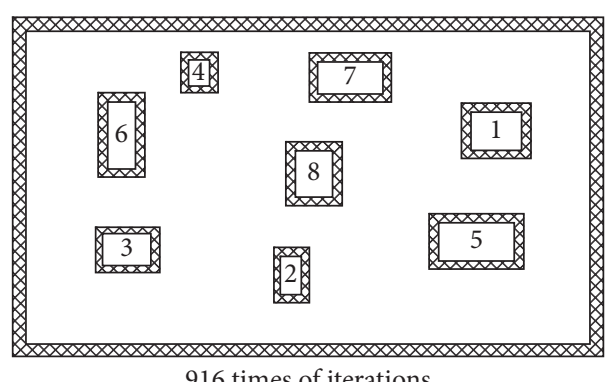

(g)

Figure 11: The layout situation of critical iterations.

Figure 2, which means that, for the ant to find a better location, the angle and distance between adjacent machines are decisive.

\section{Conclusions}

This paper presents an improved ACO based on analysis of the difference between "advantage from quantity" and "advantage from relationship," for the machine layout problem which is featured as "advantage from relationship." There exist a problem of inefficient convergence and search in bionic algorithm when used for solving MLP. Therefore, a methodology for improving ACO is formulated by considering the attached design variables under local polar coordinate. In addition, the positive feedback mechanism of pheromone perception and the inverse feedback mechanism which assigns value inversely at the end of search process significantly contribute to the improvement of ACO algorithm.

Consequently, comparative experiments are performed and the improved ACO algorithm is shown in both the 
TABLE 2: Results on different local optimal values obtained in numerous searches.

\begin{tabular}{|c|c|c|c|c|c|c|c|c|c|}
\hline \multirow[t]{2}{*}{$E$} & $x_{1}$ & $x_{2}$ & $x_{3}$ & $x_{4}$ & $x_{5}$ & $x_{6}$ & $x_{7}$ & $x_{8}$ & \multirow[t]{2}{*}{$F$} \\
\hline & $y_{1}$ & $y_{2}$ & $y_{3}$ & $y_{4}$ & $y_{5}$ & $y_{6}$ & $y_{7}$ & $y_{8}$ & \\
\hline \multirow{2}{*}{512} & 10.46 & 16.83 & 4.89 & 4.80 & 29.84 & 28.57 & 19.81 & 23.91 & \multirow{2}{*}{25036} \\
\hline & 3.40 & 9.76 & 9.74 & 17.72 & 17.54 & 5.78 & 5.23 & 13.78 & \\
\hline \multirow{2}{*}{495} & 5.73 & 12.07 & 20.56 & 26.21 & 31.50 & 31.78 & 16.92 & 4.19 & \multirow{2}{*}{25014} \\
\hline & 1.85 & 8.22 & 16.70 & 11.04 & 3.76 & 15.73 & 2.07 & 14.80 & \\
\hline \multirow{2}{*}{489} & 30.43 & 27.96 & 12.41 & 4.79 & 4.86 & 16.21 & 29.90 & 11.08 & \multirow{2}{*}{25785} \\
\hline & 9.89 & 17.49 & 1.95 & 4.41 & 13.38 & 17.12 & 2.54 & 9.31 & \\
\hline \multirow{2}{*}{518} & 31.94 & 2.98 & 27.62 & 4.21 & 10.57 & 21.03 & 31.55 & 12.73 & \multirow{2}{*}{29294} \\
\hline & 2.01 & 15.68 & 15.65 & 7.91 & 1.54 & 4.95 & 10.73 & 17.49 & \\
\hline \multirow{2}{*}{501} & 11.25 & 31.16 & 22.26 & 2.26 & 2.27 & 23.18 & 34.19 & 14.97 & \multirow{2}{*}{28826} \\
\hline & 17.73 & 8.37 & 1.88 & 1.86 & 10.85 & 17.69 & 12.93 & 7.43 & \\
\hline
\end{tabular}

E: times of iterations when sinking into the local optimal solution.

TABLE 3: Critical iterations of improved ACO algorithm under local polar coordinate systems.

\begin{tabular}{|c|c|c|c|c|c|c|c|c|c|}
\hline Generations & $\begin{array}{l}\rho_{1} \\
\theta_{1} \\
\end{array}$ & $\begin{array}{l}\rho_{2} \\
\theta_{2} \\
\end{array}$ & $\begin{array}{l}\rho_{3} \\
\theta_{3} \\
\end{array}$ & $\begin{array}{l}\rho_{4} \\
\theta_{4} \\
\end{array}$ & $\begin{array}{l}\rho_{5} \\
\theta_{5} \\
\end{array}$ & $\begin{array}{l}\rho_{6} \\
\theta_{6} \\
\end{array}$ & $\begin{array}{l}\rho_{7} \\
\theta_{7} \\
\end{array}$ & $\begin{array}{l}\rho_{8} \\
\theta_{8} \\
\end{array}$ & $F$ \\
\hline \multirow{2}{*}{100} & 29.07 & 9.01 & 11.51 & 11.37 & 15.17 & 11.19 & 15.33 & 6.10 & \multirow{2}{*}{21282} \\
\hline & 0.08 & 1.15 & 0.92 & 0.58 & 0.01 & 1.85 & 0.98 & 1.57 & \\
\hline \multirow{2}{*}{200} & 8.89 & 21.62 & 12.18 & 27.02 & 8.09 & 8.38 & 9.82 & 9.05 & \multirow{2}{*}{26883} \\
\hline & 0.05 & 0.05 & 0.44 & 1.01 & 1.32 & 1.86 & 0.31 & 1.94 & \\
\hline \multirow{2}{*}{600} & 31.01 & 9.01 & 6.72 & 9.23 & 11.27 & 12.19 & 12.12 & 7.41 & \multirow{2}{*}{20012} \\
\hline & 0.08 & 1.27 & 0.81 & 0.37 & 1.15 & 0.75 & 0.03 & 1.26 & \\
\hline \multirow{2}{*}{750} & 31.22 & 10.01 & 6.51 & 9.10 & 11.26 & 12.19 & 12.12 & 12.40 & \multirow{2}{*}{18839} \\
\hline & 0.12 & 1.38 & 0.81 & 0.37 & 1.16 & 0.74 & 1.93 & 1.47 & \\
\hline \multirow{2}{*}{800} & 28.81 & 9.01 & 5.92 & 8.30 & 11.27 & 15.09 & 12.12 & 7.40 & \multirow{2}{*}{13454} \\
\hline & 0.12 & 1.17 & 0.81 & 0.27 & 1.66 & 0.78 & 0.03 & 1.28 & \\
\hline \multirow{2}{*}{850} & 28.07 & 9.00 & 6.51 & 8.37 & 8.17 & 11.19 & 9.33 & 6.40 & \multirow{2}{*}{12542} \\
\hline & 0.08 & 1.15 & 0.82 & 0.28 & 1.65 & 0.85 & 0.13 & 1.27 & \\
\hline \multirow{2}{*}{916} & 28.81 & 9.01 & 5.92 & 8.30 & 9.27 & 12.09 & 9.12 & 6.41 & \multirow{2}{*}{11768} \\
\hline & 0.12 & 1.17 & 0.81 & 0.27 & 1.67 & 0.86 & 0.03 & 1.27 & \\
\hline
\end{tabular}

reliability of convergence and the optimization degree of solution, when solving MLP.

\section{Conflicts of Interest}

The authors declare that they have no conflicts of interest.

\section{Acknowledgments}

This research was funded by the National Natural Science Foundation of China through the project "Theory of Job Shop Reverse Scheduling Based on Second-Order Recursive Configuration for Working-Hours Allowance" (NSFC.51375357) and the ESI improvement project supported by Wuhan University of Technology.

\section{References}

[1] G. Manita, I. Chaieb, and O. Korbaa, "A new approach for loop machine layout problem integrating proximity constraints,"
International Journal of Production Research, vol. 54, no. 3, pp. 778-798, 2016.

[2] S.-W. Hou, Z. Li, and H. Wang, "A Fast Algorithm to Generate Feasible Solution of Production Facilities Layout Based on Plane Segmentation," Mathematical Problems in Engineering, vol. 2016, Article ID 1712376, 2016.

[3] M.-S. Chang and T.-C. Ku, "A slicing tree representation and QCP-model-based heuristic algorithm for the unequal-area block facility layout problem," Mathematical Problems in Engineering, vol. 2013, Article ID 853586, 2013.

[4] X. Liu, H. Yi, and Z. Ni, "Application of ant colony optimization algorithm in process planning optimization," Journal of Intelligent Manufacturing, vol. 24, no. 1, pp. 1-13, 2013.

[5] H. Samarghandi and K. Eshghi, "An efficient tabu algorithm for the single row facility layout problem," European Journal of Operational Research, vol. 205, no. 1, pp. 98-105, 2010.

[6] S. Selvakumar, K. P. Arulshri, K. P. Padmanaban, and K. S. K. Sasikumar, "Design and optimization of machining fixture layout using ANN and DOE," The International Journal of 
Advanced Manufacturing Technology, vol. 65, no. 9-12, pp. 15731586, 2013.

[7] D. Datta, A. R. Amaral, and J. R. Figueira, "Single row facility layout problem using a permutation-based genetic algorithm," European Journal of Operational Research, vol. 213, no. 2, pp. 388-394, 2011.

[8] Y. Luo, G. Feng, F. Zhang, Y. Mao, and J. Wu, "A reverse constraint satisfying method for earliness/tardiness scheduling problem based on improved genetic algorithm," Journal of Computational Methods in Sciences and Engineering, vol. 15, no. 3, pp. 515-525, 2015.

[9] M. E. Aydin, "Coordinating metaheuristic agents with swarm intelligence," Journal of Intelligent Manufacturing, vol. 23, no. 4, pp. 991-999, 2012.

[10] I. Brajevic and M. Tuba, "An upgraded artificial bee colony (ABC) algorithm for constrained optimization problems," Journal of Intelligent Manufacturing, vol. 24, no. 4, pp. 729-740, 2013.

[11] B. Akay and D. Karaboga, "Artificial bee colony algorithm for large-scale problems and engineering design optimization," Journal of Intelligent Manufacturing, vol. 23, no. 4, pp. 1001-1014, 2012.

[12] S. Klancnik, M. Brezocnik, J. Balic, and I. Karabegovic, "Programming of $\mathrm{CNC}$ milling machines using particle swarm optimization," Materials and Manufacturing Processes, vol. 28, no. 7, pp. 811-815, 2013.

[13] B. Akay, "A study on particle swarm optimization and artificial bee colony algorithms for multilevel thresholding," Applied Soft Computing Journal, vol. 13, no. 6, pp. 3066-3091, 2013.

[14] Y. Luo, "Topological sorting-based two-stage nested ant colony algorithm for job-shop scheduling problem," Jixie Gongcheng Xuebao/Journal of Mechanical Engineering, vol. 51, no. 8, pp. 178-184, 2015.

[15] Y. Luo, "Nested optimization method combining complex method and ant colony optimization to solve JSSP with complex associated processes," Journal of Intelligent Manufacturing, 2015.

[16] J.-P. Arnaout, "Ant colony optimization algorithm for the Euclidean location-allocation problem with unknown number of facilities," Journal of Intelligent Manufacturing, vol. 24, no. 1, pp. $45-54,2013$.

[17] B. H. Ulutas and S. Kulturel-Konak, "Assessing hypermutation operators of a clonal selection algorithm for the unequal area facility layout problem," Engineering Optimization, vol. 45, no. 3, pp. 375-395, 2013.

[18] P. Hungerländer and F. Rendl, "A computational study and survey of methods for the single-row facility layout problem," Computational Optimization and Applications. An International Journal, vol. 55, no. 1, pp. 1-20, 2013.

[19] A. R. Amaral, "Optimal solutions for the double row layout problem," Optimization Letters, vol. 7, no. 2, pp. 407-413, 2013.

[20] G. Moslemipour and T. S. Lee, "Intelligent design of a dynamic machine layout in uncertain environment of flexible manufacturing systems," Journal of Intelligent Manufacturing, vol. 23, no. 5, pp. 1849-1860, 2012.

[21] A. Sadrzadeh, "A genetic algorithm with the heuristic procedure to solve the multi-line layout problem," Computers \& Industrial Engineering, vol. 62, no. 4, pp. 1055-1064, 2012.

[22] K. Chandrasekar and P. Venkumar, "Genetic algorithm approach for integrating cell formation with machine layout and cell layout," International Journal of Operational Research, vol. 16, no. 2, pp. 155-171, 2013.
[23] A. Drira, H. Pierreval, and S. Hajri-Gabouj, "Design of a robust layout with information uncertainty increasing over time: A fuzzy evolutionaryapproach," Engineering Applications of Artificial Intelligence, vol. 26, no. 3, pp. 1052-1060, 2013.

[24] M. Saravanan and P. V. Arulkumar, "Design and optimisation for fixed area cellular layout problems using GA and SAA," International Journal of Innovation and Sustainable Development, vol. 7, no. 1, pp. 91-109, 2013.

[25] Y. Luo, "Simulation Experiment Exploration of Genetic Algorithm's Convergence over the Relationship Advantage Problem," Mathematical Problems in Engineering, vol. 2016, Article ID 4527402, 2016. 


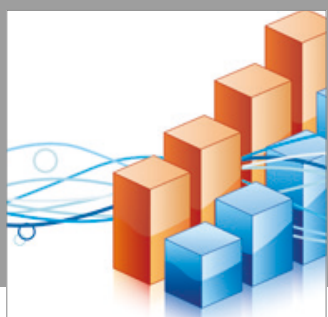

Advances in

Operations Research

vatersals

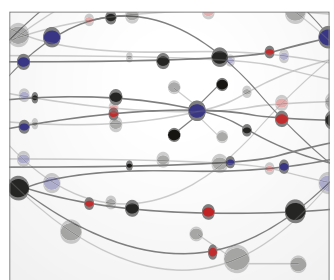

\section{The Scientific} World Journal
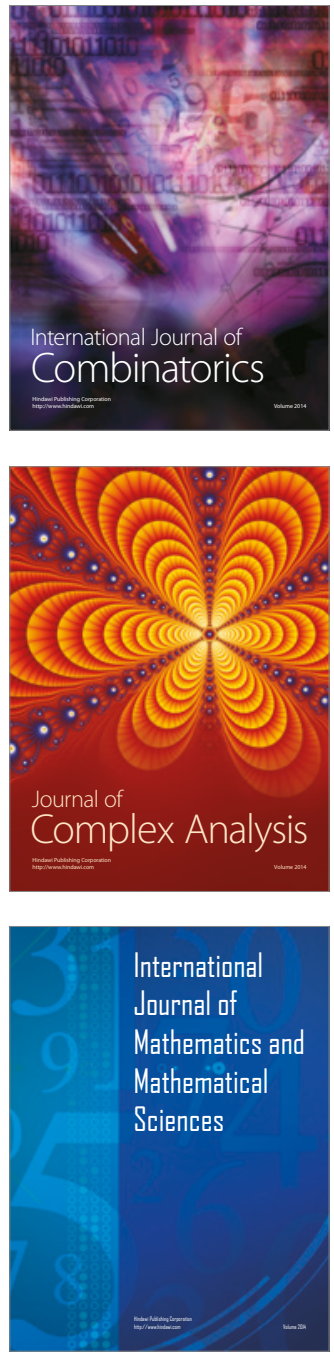
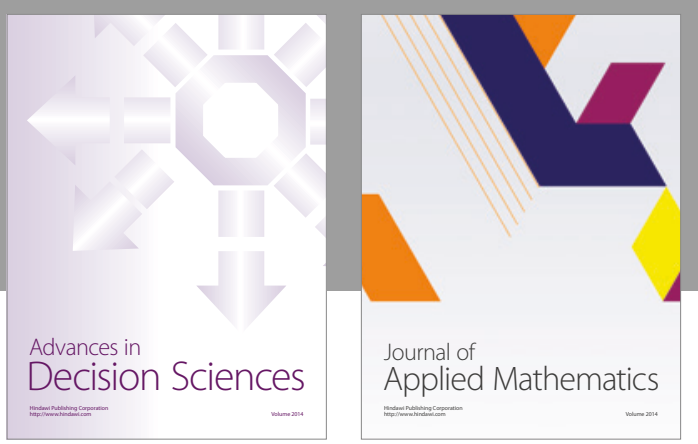

Algebra

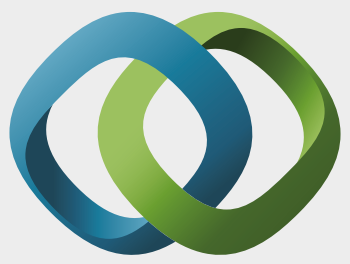

\section{Hindawi}

Submit your manuscripts at

https://www.hindawi.com
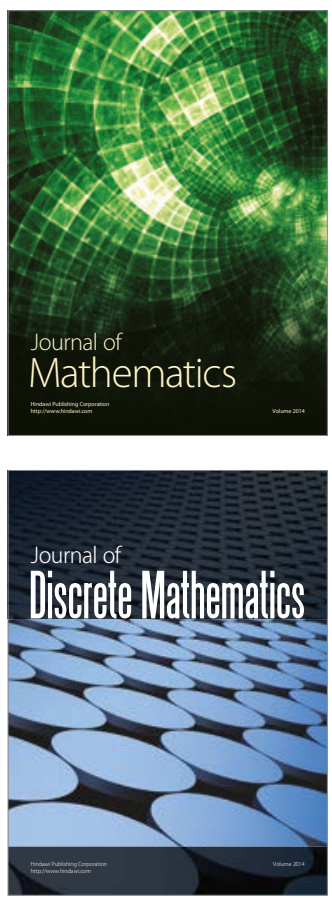

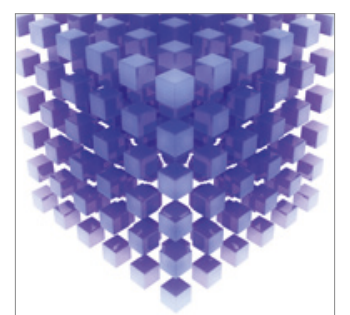

Mathematical Problems in Engineering
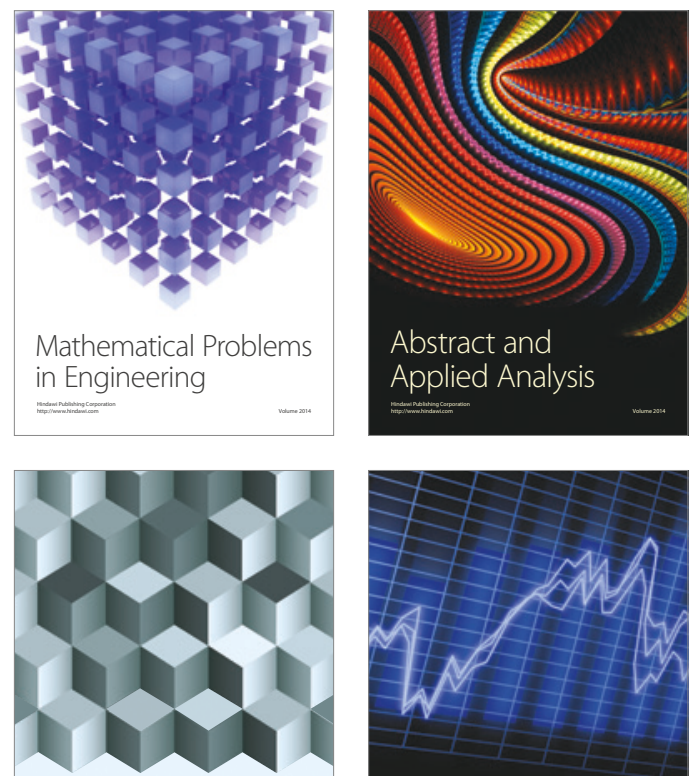

Journal of

Function Spaces

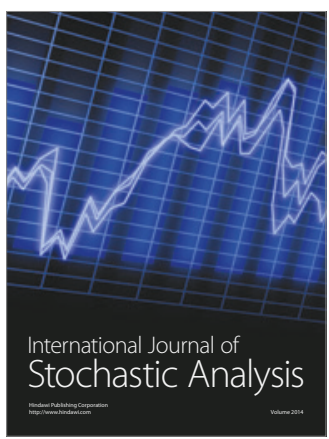

Probability and Statistics
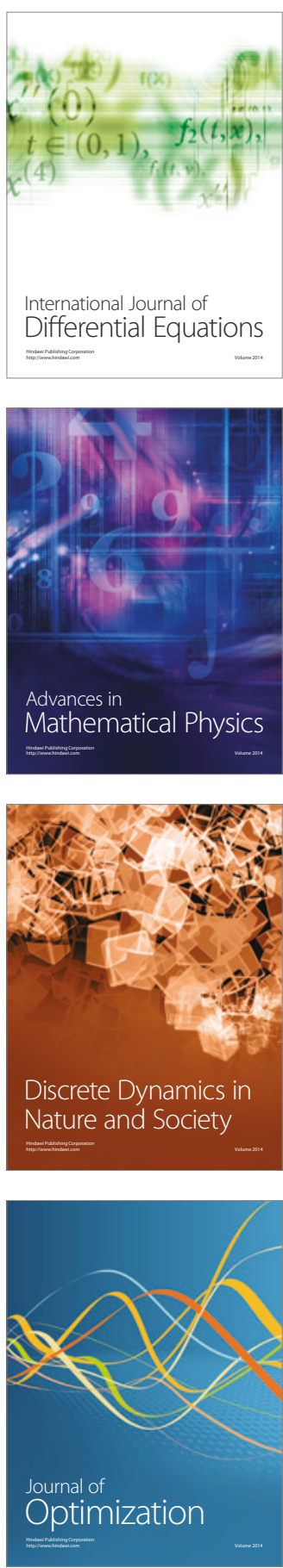